\title{
ANALYTIC NETWORK PROCESS AS QUALITATIVE SIMULATING TOOL: RESEARCHING OF FINANCIAL CRISIS
}

\author{
Olga Andreichicova* \\ Moscow Institute of Physics and Technology \\ Moscow, Russia \\ E-mail: olga_andr@hotmail.com \\ Alexander Andreichicov \\ Russian State University of Innovation Technology and Business \\ Moscow, Russia \\ E-mail: alexandrol@mail.ru
}

\begin{abstract}
Analytic Network Process is the convenient tool for simulating of complex problems under uncertainty. Network model describes the problem by the qualitative attributes such as clusters, nodes and relations between them. These attributes can present essential factors, criteria, properties, functions, actors and other aspects of the considered problem. The researcher also can use quantitative data in the form of expert judgments for experimental evaluating of mutual influences of the attributes. Thus we have an opportunity to explore complex problem and to obtain unobvious results by experimenting with model and to achieve the better understanding of the problem for decision making. We show the example, which demonstrates some capabilities of ANP for the researching of the efficiency of the measures for the surmounting of financial crisis.
\end{abstract}

Keywords: ANP, simulating, priorities, financial crisis

\section{Introduction}

Analytic Network Process (ANP) developed by Thomas Saaty (Saaty, 2001) is a powerful tool for system analysis and qualitative simulating. One of its main advantages is that it can be applied under uncertainty, when other techniques do not work. Network model describes the problem by the qualitative attributes such as clusters, nodes and relations between them. These attributes can present essential factors, criteria, properties, functions, actors and other aspects of the considered problem. We also can use quantitative data in the form of expert judgments for experimental evaluating of mutual influences of the attributes. Thus we have an opportunity to explore complex problem and to obtain unobvious results by experimenting with model and to achieve the better understanding of the problem for decision making.

In this paper we are researching the problem of financial crisis with use of ANP. We would like to find out, what measures should take the governments to cope with recession, unemployment and other consequences of the crisis. How these measures will influence main economic indicators and what of them have the greater effect. We don't purport any specific country in this example and take into consideration economic aspects and relations, which are universal for every state.

\footnotetext{
${ }^{*}$ Corresponding author
} 


\section{Network model}

At first, we shall build the network model to learn, how the set of measures, which many governments are taking now, will influence main economic indicators. We take into consideration the set of attributes, joint into following clusters:

\subsection{Government}

This cluster contains 6 elements (nodes) that present the measures, which government can take for surmounting of the crisis. They are:

- Export Incentive. For example, the government can devaluate exchange course of national currency to stimulate export or furnish a financial assistance exporters or use political tools in foreign-economic activity.

- Financial Support. So we name direct financial assistance of the government to banks, producers and population.

- Interest Rate. Many states have lowered the refinancing rate to support crediting, but some countries have marked up it.

- Poor Assets' Purchase. The governments purchase the poor assets of the banks and producers to avoid chain reaction and to keep population from poverty.

- Protectionism. This is meant the imposing of custom duties for import goods to raise demand for national production.

- $\quad$ Tax Cut. The government can reduce taxes for population and producers for hard time.

\subsection{Banks}

Banks were the sources of financial crisis. They are main actors in economics, which supplies with money the producers and population. We include 4 following nodes into this cluster:

- Assets. The assets of many banks strongly change during the crisis. Undoubtedly, banks' assets influence all kinds of their activity.

- Credits. This is main function of a bank, which need not comments.

- Deposits. Financial difficulties have influence on bank deposits, so this node is important for our research.

- Mortgage. World financial crisis has begun with mortgage crisis in USA, therefore this factor should be taken into account.

\subsection{Producers}

Main goal for world economics is to overcome the recession. In view of this goal we consider the following attributes of producers:

- Capitalization. The drop of stock market and companies' value influence production volume, employment and other important economic parameters, so it is included in our model.

- Innovation. Along with difficulties the crisis creates incentives for innovation, because producers have to find more effective technologies and to make more procurable and more attractive goods for consumers. Innovation can save some producers and create new markets.

- Output. So we name production volume, which is one of the most important characteristics of any producer.

- Profit. This attribute does not need comments.

\subsection{Science and Technologies}

Science is the base of new technologies and innovations. Recession compels producers to find help in science researches. The governments usually do not reduce investments into scientific researches in hard time. We consider 2 main nodes in this cluster: 
- Costs. Governments or producers can change the costs for scientific researches during the crisis, what in turn can influence new technologies, innovation and other important factors.

- New Technologies are main outcomes of scientific researches.

\subsection{Population}

Population is undergone the negative impact of financial crisis such as loss of jobs, reduction in incomes and others. In turn, people forms demand for goods and credits, their trust influences bank deposits. We include the following obvious elements in this cluster:

- Demand.

- Incomes.

- Trust.

\subsection{Macrofactors}

This cluster contains main economic indicators that should be improved in result of government's measures:

- Capital Investments.

- Employment.

- Inflation.

- Market Shares.

- Production Level.

We think these indicators don't need a comment, except that someone could consider another set, but every researcher has a right to form own network model and to substantiate it at that. Network model is shown in Figure 1, where arrows between the clusters denominate the influence directions. We have used the SuperDecisions software (SuperDecisions, 2009) for the ANP in our research.

Note this model is assigned for evaluating positive consequences of the measures, being taken by the government. It is important, as many influences can have both positive and negative direction. Here we are researching only positive influences. If someone would like to take into consideration negative influence of these or other factors, he should build a model (its structure may stay the same) for the revealing their impact. It should not to mix positive and negative influences in one example, because this can bring to false outcomes. We can research in one example positive influences and in other example negative ones. After that we can obtain integrated estimation of influence with use Benefit - Cost $(B O)$ approach, implying positive influences as benefits and negative as costs.

When we were evaluating the positive influence of clusters and elements (nodes), we wanted to learn, how crisis management measures, have been taken by the government, would influence banks, producers, population, science and macrofactors. Comparing the clusters and nodes, we asked "What of two compared elements (clusters) the analyzed element (cluster) influence more and how much more?" At that experts filled pair comparison matrixes, like the following:

\begin{tabular}{c|ccccc|c}
$\begin{array}{c}\text { Comparing by } \\
\text { the cluster } \\
\text { Banks }\end{array}$ & Banks & Producers & Population & Sc\&Tech & Macrofactors & $w$ \\
\hline Banks & 1 & 2 & 3 & 9 & 5 & 0,4574 \\
Producers & & 1 & 2 & 5 & 3 & 0,087 \\
Population & & & 1 & 3 & 2 & 0,1497 \\
Sc\&Tech & & & & 1 & $1 / 2$ & 0,2572 \\
Macrofactors & & & & & 1 & 0,0487
\end{tabular}

Priorities of influence for all clusters are brought in Table1 in Appendix. 


\begin{tabular}{c|cccc|c}
$\begin{array}{c}\text { Comparing nodes of cluster } \\
\text { Macrofactors by the node } \\
\text { Innovation }\end{array}$ & $\begin{array}{c}\text { Capital } \\
\text { Investments }\end{array}$ & Employment & $\begin{array}{c}\text { Market } \\
\text { Shares }\end{array}$ & $\begin{array}{c}\text { Production } \\
\text { Level }\end{array}$ & $\boldsymbol{w}$ \\
\hline Capital Investments & 1 & 1 & $1 / 2$ & $1 / 4$ & 0,125 \\
Employment & & 1 & $1 / 2$ & $1 / 4$ & 0,125 \\
Market Shares & & & 1 & $1 / 2$ & 0,25 \\
Production Level & & & & 1 & 0,5
\end{tabular}

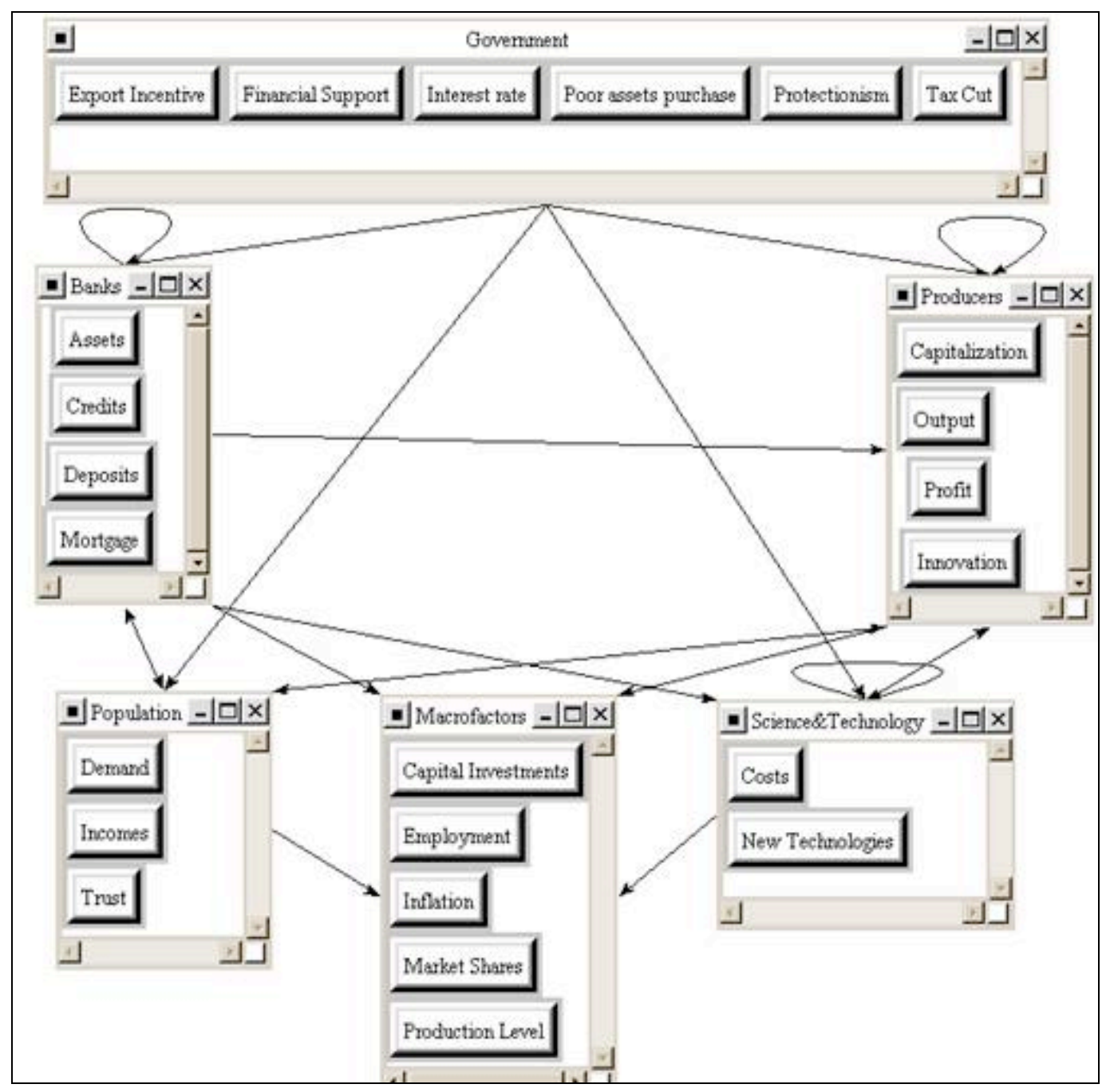

Figure 1. Network model for evaluating of positive influence.

Priorities of influence for all nodes of the network model are brought in unweighted supermatrix in Table 2 in Appendix. In Table 3 the weighted supermatrix is shown, and limiting priorities are brought in Figure 2 and Figure 3. We can see that cluster Producers is the most influenced by other clusters in the model, then cluster Macrofactors follows.

In Table 4 are brought limiting priorities of clusters and nodes, normalized by cluster. Influences, which our model describes, have produced the greatest impact upon the nodes of the cluster Producers, and this testifies that government's measures are correct, if they will be properly put into practice. We are interested in limiting priorities of Macrofactor's nodes at existing influences. We see that the greatest positive 
effect will have Production Level, Employment will be improved too. Other indicators will become better in a less degree. The mutual influences of the considered factors bring to positive impact on Science and Technologies, Banks (especially Assets), Demand and Trust (in cluster Population). These results are consistent with well known economic regularities.

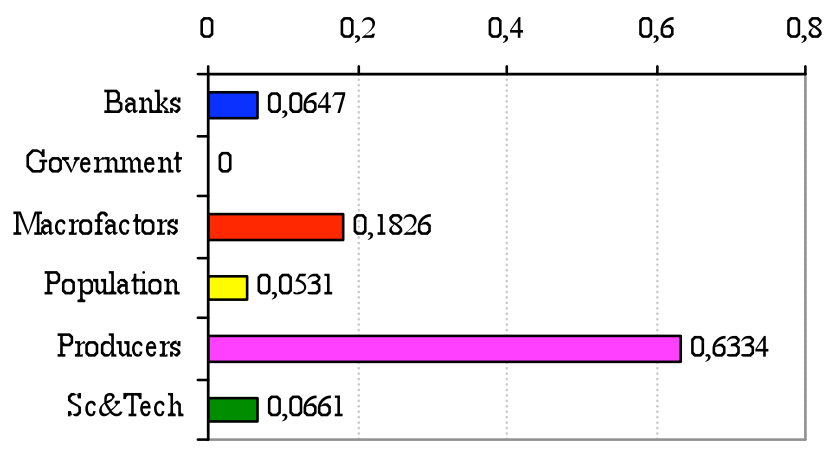

Figure 2. Limiting priorities of the clusters.

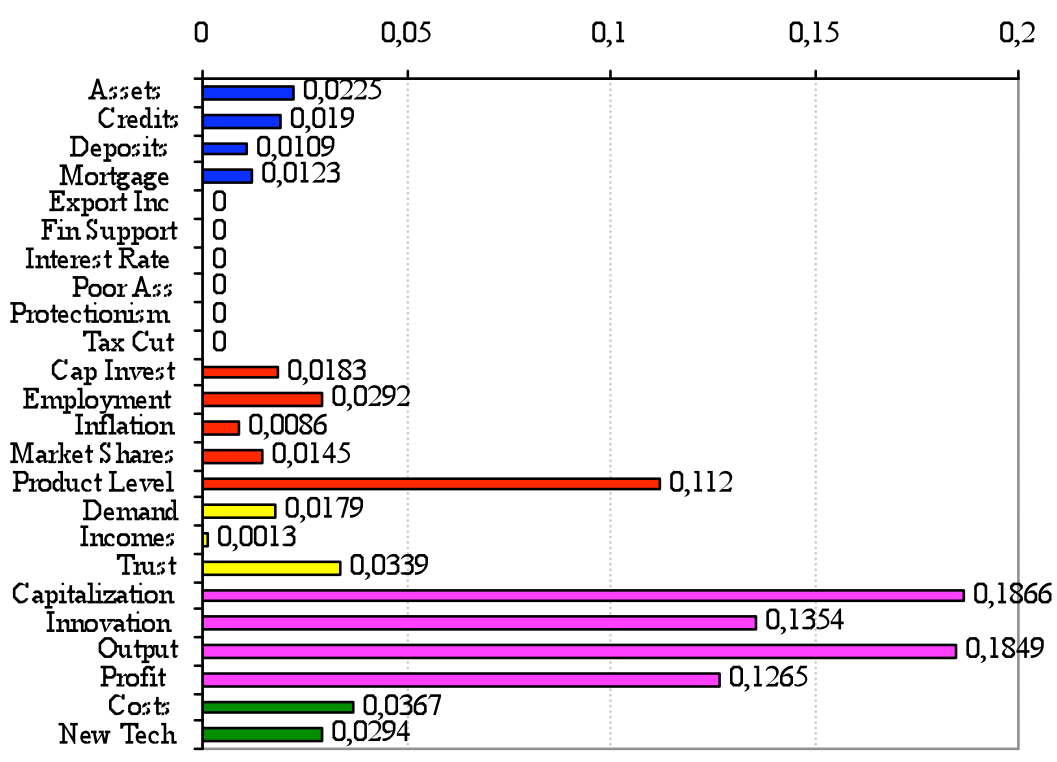

Figure 3. Limiting priorities of the nodes.

Note that cluster Government is a source in network, therefore limiting priorities of its nodes are zero. Moreover, if this cluster will be removed from network, then limiting priorities of remaining nodes will not change. The modified network model shown in Figure 4 gives the same limiting priorities of all nodes. These outcomes are brought in Table 4. 


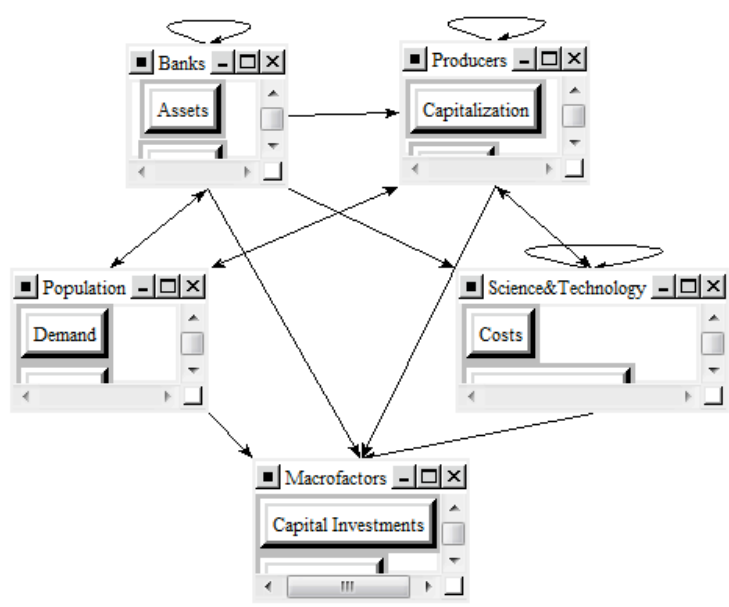

Figure 4. Modified network model.

\section{Inverted network}

We have satisfied that limiting priorities of Macrofactors and other clusters do not depend on clusterssources provided all expert judgments are constant. But we are interested, what of the Government's nodes are more influential? To answer this question we have inverted our network model and consider the inverted task. The structure of this task is shown in Figure 5.

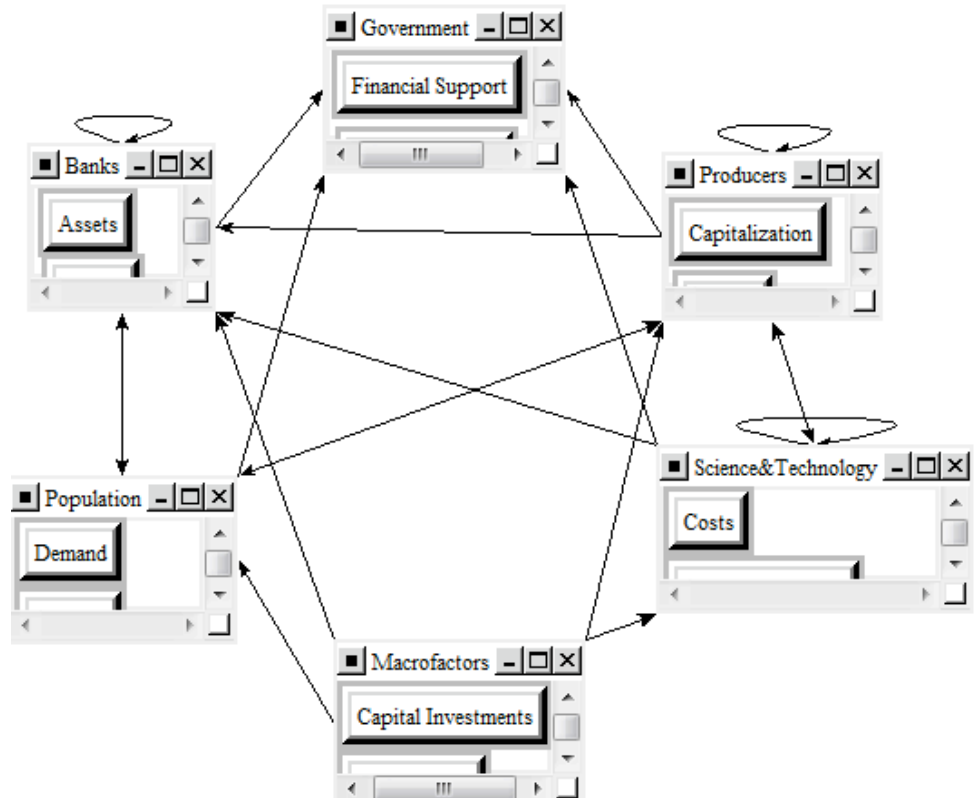

Figure 5. Network model for the inverted task.

When filling pair comparison matrixes, experts should answer the questions "What of two compared clusters (nodes) influence the analyzed cluster (node) more, and how much more?" Priorities calculated for all matrixes are brought in unweighted supermatrix (see Table 5). There is the weighted supermatrix in Table 6 and cluster matrix in Table 7. Limiting priorities of the nodes and clusters are shown in Table 4 and in Figure 6, 7 . 


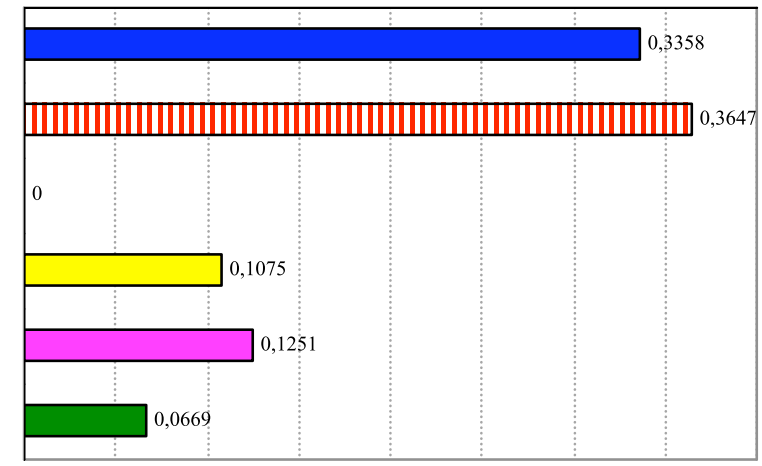

Figure 6. Limiting priorities of the clusters for the inverted task.

We can see that cluster Government is the most influential, after that the cluster Banks follows. Impact of clusters Producers and Population is considerably less. The most influential node is Financial Support, Poor Assets' Purchase is at the second place, Protectionism takes the latter place. Among the elements of the cluster Banks the most influential are Mortgage and Credits. Note, that Innovation is the most important element in the cluster Producers. New Technologies (cluster Science and Technologies), Demand and Incomes (cluster Population) have close limiting priorities.

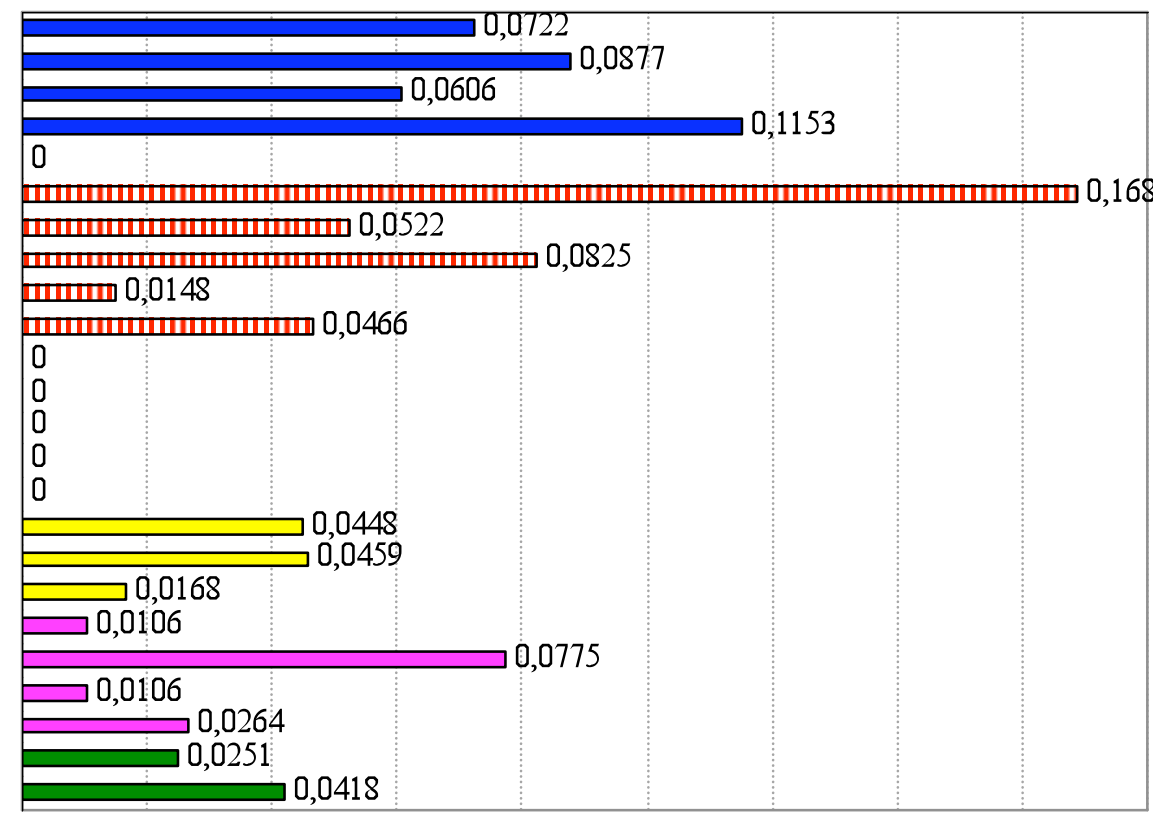

Figure 7. Limiting priorities of the nodes in inverted task.

The results obtained can help to understand, what consequences different measures can bring to. These outcomes are reasonable and correspond to real facts at the moment. Priorities of the measures and inten- 
sities of influences can differ for different counties and can change in time. Researchers can change their preferences and the sets of considered elements. We hope this example demonstrate that ANP is very useful tool for system analysis and decision making.

\section{APPENDIX}

Table 1. Cluster matrix for network model.

\begin{tabular}{|c|c|c|c|c|c|c|}
\hline $\begin{array}{c}\text { Cluster } \\
\text { Name }\end{array}$ & Banks & Government & Macrofactors & Population & Producers & Sc \& Tech \\
\hline Banks & 0,4574 & 0,25 & 0 & 0,2857 & 0 & 0 \\
\hline Government & 0 & 0 & 0 & 0 & 0 & 0 \\
\hline Macrofactors & 0,087 & 0 & 0 & 0,1429 & 0,1409 & 0,1005 \\
\hline Population & 0,1497 & 0,25 & 0 & 0 & 0,2628 & 0 \\
\hline Producers & 0,2572 & 0,25 & 0 & 0,5714 & 0,4554 & 0,4331 \\
\hline Sc \& Tech & 0,0487 & 0,25 & 0 & 0 & 0,1409 & 0,4664
\end{tabular}


Table 2. Unweighted supermatrix for the network model.

\begin{tabular}{|c|c|c|c|c|c|c|c|c|c|c|c|c|c|c|c|c|c|c|c|c|c|c|c|c|}
\hline & $\begin{array}{l}n \\
0 \\
0 \\
0 \\
2\end{array}$ & 总 & 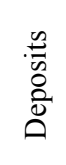 & 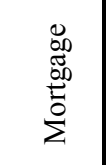 & 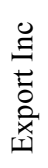 & 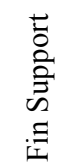 & 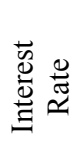 & 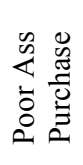 & 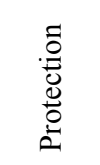 & 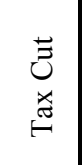 & 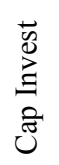 & $\underset{\text { 四 }}{\stackrel{\Xi}{\Xi}}$ & 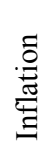 & 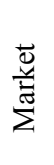 & 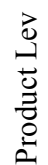 & 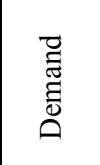 & 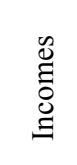 & $\stackrel{\vec{n}}{E}$ & 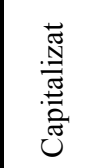 & 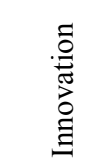 & 言 & $\begin{array}{l}\overrightarrow{0} \\
\text { D. } \\
\text { D }\end{array}$ & 岂 & $\begin{array}{l}\frac{1}{0} \\
\stackrel{0}{\Theta} \\
3 \\
0 \\
z\end{array}$ \\
\hline Assets & 0 & 1 & 0,6 & 0,667 & 0 & 0 & 0 & 1 & 0 & 0 & 0 & 0 & 0 & 0 & 0 & 0 & 0 & 0 & 0 & 0 & 0 & 0 & 0 & 0 \\
\hline Credits & 0,5714 & 0 & 0,2 & 0,2222 & 1 & 0,5 & 0,75 & 0 & 0 & 0 & 0 & 0 & 0 & 0 & 0 & 0,6667 & 0,25 & 0,1634 & 0 & 0 & 0 & 0 & 0 & 0 \\
\hline Deposits & 0,1429 & 0 & 0 & 0,1111 & 0 & 0 & 0 & 0 & 0 & 0 & 0 & 0 & 0 & 0 & 0 & 0 & 0,5 & 0,5396 & 0 & 0 & 0 & 0 & 0 & 0 \\
\hline Mortgage & 0,2857 & 0 & 0,2 & 0 & 0 & 0,5 & 0,25 & 0 & 0 & 0 & 0 & 0 & 0 & 0 & 0 & 0,3333 & 0,25 & 0,297 & 0 & 0 & 0 & 0 & 0 & 0 \\
\hline $\begin{array}{l}\text { Export } \\
\text { Inc }\end{array}$ & 0 & 0 & 0 & 0 & 0 & 0 & 0 & 0 & 0 & 0 & 0 & 0 & 0 & 0 & 0 & 0 & 0 & 0 & 0 & 0 & 0 & 0 & 0 & 0 \\
\hline Fin Sup & 0 & 0 & 0 & 0 & 0 & 0 & 0 & 0 & 0 & 0 & 0 & 0 & 0 & 0 & 0 & 0 & 0 & 0 & 0 & 0 & 0 & 0 & 0 & 0 \\
\hline Interest & 0 & 0 & 0 & 0 & 0 & 0 & 0 & 0 & 0 & 0 & 0 & 0 & 0 & 0 & 0 & 0 & 0 & 0 & 0 & 0 & 0 & 0 & 0 & 0 \\
\hline Poor Ass & 0 & 0 & 0 & 0 & 0 & 0 & 0 & 0 & 0 & 0 & 0 & 0 & 0 & 0 & 0 & 0 & 0 & 0 & 0 & 0 & 0 & 0 & 0 & 0 \\
\hline Protect & 0 & 0 & 0 & 0 & 0 & 0 & 0 & 0 & 0 & 0 & 0 & 0 & 0 & 0 & 0 & 0 & 0 & 0 & 0 & 0 & 0 & 0 & 0 & 0 \\
\hline Tax Cut & 0 & 0 & 0 & 0 & 0 & 0 & 0 & 0 & 0 & 0 & 0 & 0 & 0 & 0 & 0 & 0 & 0 & 0 & 0 & 0 & 0 & 0 & 0 & 0 \\
\hline $\begin{array}{l}\text { Cap } \\
\text { Invest }\end{array}$ & 0,75 & 0 & 0,6 & 0,75 & 0 & 0 & 0 & 0 & 0 & 0 & 0 & 0 & 0 & 0 & 0 & 0 & 0 & 0 & 0 & 0,125 & 0 & 0,25 & 0 & 0,4804 \\
\hline Employm & 0 & 0 & 0 & 0 & 0 & 0 & 0 & 0 & 0 & 0 & 0 & 0 & 0 & 0 & 0 & 0 & 0 & 0 & 0 & 0,125 & 0,4778 & 0 & 0 & 0,2158 \\
\hline Inflation & 0 & 0,2 & 0,2 & 0,25 & 0 & 0 & 0 & 0 & 0 & 0 & 0 & 0 & 0 & 0 & 0 & 0,1429 & 0,2 & 0 & 0 & 0 & 0,1281 & 0 & 0 & 0 \\
\hline Market & 0 & 0 & 0 & 0 & 0 & 0 & 0 & 0 & 0 & 0 & 0 & 0 & 0 & 0 & 0 & 0,2857 & 0,2 & 0 & 0 & 0,25 & 0,138 & 0 & 0 & 0,1079 \\
\hline Prod Lev & 0,25 & 0,8 & 0,2 & 0 & 0 & 0 & 0 & 0 & 0 & 0 & 0 & 0 & 0 & 0 & 0 & 0,5714 & 0,6 & 0 & 1 & 0,5 & 0,2561 & 0,75 & 0 & 0,1959 \\
\hline Demand & 0 & 0,6 & 0 & 0,4 & 0 & 0 & 0 & 0 & 0 & 0 & 0 & 0 & 0 & 0 & 0 & 0 & 0 & 0 & 0 & 0,3333 & 0 & 0 & 0 & 0 \\
\hline Incomes & 0 & 0,2 & 0 & 0,2 & 0 & 0 & 0 & 0 & 0 & 0,5 & 0 & 0 & 0 & 0 & 0 & 0 & 0 & 0 & 0 & 0 & 0 & 0 & 0 & 0 \\
\hline Trust & 0 & 0,2 & 1 & 0,4 & 0 & 0 & 0 & 0 & 0 & 0,5 & 0 & 0 & 0 & 0 & 0 & 0 & 0 & 0 & 0 & 0,6667 & 0 & 0 & 0 & 0 \\
\hline Capitaliz & 0 & 0 & 0 & 0 & 0 & 0 & 0 & 1 & 0 & 0 & 0 & 0 & 0 & 0 & 0 & 0,1516 & 0 & 1 & 0 & 0,5 & 0,3 & & & \\
\hline Innovat & 0,3333 & 0,3333 & 0 & 0 & 0 & 0,5 & 0 & 0 & 0,3333 & 0,25 & 0 & 0 & 0 & 0 & 0 & 0,0758 & 0,2 & 0 & 0,3333 & 0 & 0,1 & 0,143 & 1 & 1 \\
\hline Output & 0,6667 & 0,6667 & 0 & 0 & 0 & 0,5 & 0 & 0 & 0,6667 & 0 & 0 & 0 & 0 & 0 & 0 & 0,49 & 0,4 & 0 & 0,6667 & $7 \quad 0,25$ & 0 & 0,2857 & 0 & 0 \\
\hline Profit & 0 & 0 & 0 & 0 & 0 & 0 & 0 & 0 & 0 & 0,75 & 0 & 0 & 0 & 0 & 0 & 0,2828 & 0,4 & 0 & 0 & 0,25 & 0,6 & 0 & 0 & 0 \\
\hline $\begin{array}{l}\text { Costs } \\
\text { New }\end{array}$ & 1 & 0,75 & 0 & 0 & 0 & 0,25 & 0 & 0 & 0 & 0 & 0 & 0 & 0 & 0 & 0 & 0 & 0 & 0 & 0 & 0,75 & 0 & 0 & 0 & 1 \\
\hline Tech & 0 & 0,25 & 0 & 0 & 0 & 0,75 & 0 & 0 & 0 & 1 & 0 & 0 & 0 & 0 & 0 & 0 & 0 & 0 & 0 & 0,25 & 0 & 0 & 1 & 0 \\
\hline
\end{tabular}


Table 3. Weighted supermatrix for the network model.

\begin{tabular}{|c|c|c|c|c|c|c|c|c|c|c|c|c|c|c|c|c|c|c|c|c|c|c|c|c|}
\hline & $\begin{array}{l}0 \\
0 \\
0 \\
0 \\
0\end{array}$ & 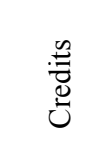 & $\begin{array}{l}\stackrel{\mathscr{U}}{0} \\
0 \\
0 \\
0\end{array}$ & 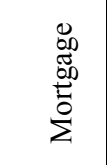 & 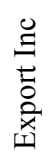 & 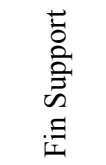 & 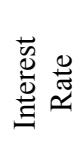 & 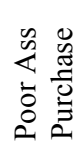 & 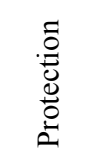 & 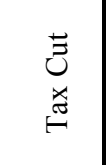 & 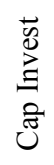 & 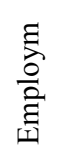 & $\begin{array}{l}\stackrel{\Xi}{\Xi} \\
\stackrel{\Xi}{\leftrightarrows} \\
\Xi\end{array}$ & $\begin{array}{l}\frac{\vec{v}}{\grave{y}} \\
\stackrel{\vec{J}}{\Sigma}\end{array}$ & 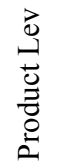 & 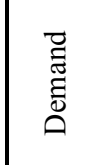 & 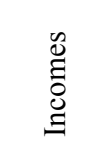 & $\stackrel{\overrightarrow{\underline{T}}}{\Xi}$ & 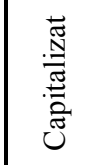 & 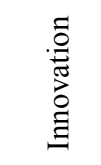 & 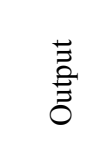 & 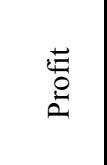 & $\begin{array}{l}0 \\
0 \\
0 \\
0\end{array}$ & $\begin{array}{l}\text { J } \\
\stackrel{0}{0} \\
\vec{z} \\
z\end{array}$ \\
\hline Assets & 0 & 0,4574 & 0,3954 & + 0,4393 & 0 & 0 & 0 & 0,5 & 0 & 0 & 0 & 0 & 0 & 0 & 0 & 0 & 0 & 0 & 0 & 0 & 0 & 0 & 0 & 0 \\
\hline Credits & 0,3074 & 0 & 0,1318 & 0,1464 & 1 & 0,1667 & 0,75 & 0 & 0 & 0 & 0 & 0 & 0 & 0 & 0 & 0,1905 & 0,0714 & 0,055 & 0 & 0 & 0 & 0 & 0 & 0 \\
\hline Deposits & 0,0769 & 0 & 0 & 0,0732 & 0 & 0 & 0 & 0 & 0 & 0 & 0 & 0 & 0 & 0 & 0 & 0 & 0,1429 & 0,178 & 0 & 0 & 0 & 0 & 0 & 0 \\
\hline Mortgage & 0,1537 & 0 & 0,1318 & 0 & 0 & 0,1667 & 0,25 & 0 & 0 & 0 & 0 & 0 & 0 & 0 & 0 & 0,0952 & 0,0714 & 0,1 & 0 & 0 & 0 & 0 & 0 & 0 \\
\hline $\begin{array}{l}\text { Export } \\
\text { Inc }\end{array}$ & 0 & 0 & 0 & 0 & 0 & 0 & 0 & 0 & 0 & 0 & 0 & 0 & 0 & 0 & 0 & 0 & 0 & 0 & 0 & 0 & 0 & 0 & 0 & 0 \\
\hline Fin Sup & 0 & 0 & 0 & 0 & 0 & 0 & 0 & 0 & 0 & 0 & 0 & 0 & 0 & 0 & 0 & 0 & 0 & 0 & 0 & 0 & 0 & 0 & 0 & 0 \\
\hline Interest & 0 & 0 & 0 & 0 & 0 & 0 & 0 & 0 & 0 & 0 & 0 & 0 & 0 & 0 & 0 & 0 & 0 & 0 & 0 & 0 & 0 & 0 & 0 & 0 \\
\hline Poor Ass & 0 & 0 & 0 & 0 & 0 & 0 & 0 & 0 & 0 & 0 & 0 & 0 & 0 & 0 & 0 & 0 & 0 & 0 & 0 & 0 & 0 & 0 & 0 & 0 \\
\hline Protect & 0 & 0 & 0 & 0 & 0 & 0 & 0 & 0 & 0 & 0 & 0 & 0 & 0 & 0 & 0 & 0 & 0 & 0 & 0 & 0 & 0 & 0 & 0 & 0 \\
\hline Tax Cut & 0 & 0 & 0 & 0 & 0 & 0 & 0 & 0 & 0 & 0 & 0 & 0 & 0 & 0 & 0 & 0 & 0 & 0 & 0 & 0 & 0 & 0 & 0 & 0 \\
\hline $\begin{array}{l}\text { Cap } \\
\text { Invest }\end{array}$ & 0,0768 & 0 & 0,0752 & 20,094 & 0 & 0 & 0 & 0 & 0 & 0 & 0 & 0 & 0 & 0 & 0 & 0 & 0 & 0 & 0 & 0,0176 & 0 & 0,0591 & 0 & 0,0483 \\
\hline Employ & 0 & 0 & 0 & 0 & 0 & 0 & 0 & 0 & 0 & 0 & 0 & 0 & 0 & 0 & 0 & 0 & 0 & 0 & 0 & 0,0176 & 60,1129 & 0 & 0 & 0,0217 \\
\hline Inflation & 0 & 0,0174 & 0,0251 & 0,0313 & 0 & 0 & 0 & 0 & 0 & 0 & 0 & 0 & 0 & 0 & 0 & 0,0204 & 0,0286 & 0 & 0 & 0 & 0,0303 & 0 & 0 & 0 \\
\hline Market & 0 & 0 & 0 & 0 & 0 & 0 & 0 & 0 & 0 & 0 & 0 & 0 & 0 & 0 & 0 & 0,0408 & 0,0286 & 0 & 0 & 0,0352 & 0,0326 & 0 & 0 & 0,0109 \\
\hline Prod Lev & 0,0256 & 0,0696 & 0,0251 & 0 & 0 & 0 & 0 & 0 & 0 & 0 & 0 & 0 & 0 & 0 & 0 & 0,0816 & 0,0857 & 0 & 0,2363 & 0,0704 & $+0,0605$ & 0,1772 & 0 & 0,0197 \\
\hline Demand & 0 & 0,0898 & 0 & 0,0863 & 0 & 0 & 0 & 0 & 0 & 0 & 0 & 0 & 0 & 0 & 0 & 0 & 0 & 0 & 0 & 0,0876 & 0 & 0 & 0 & 0 \\
\hline Incomes & 0 & 0,0299 & 0 & 0,0431 & 0 & 0 & 0 & 0 & 0 & 0,1667 & 0 & 0 & 0 & 0 & 0 & 0 & 0 & 0 & 0 & 0 & 0 & 0 & 0 & 0 \\
\hline Trust & 0 & 0,0299 & 0,2157 & 70,0863 & 0 & 0 & 0 & 0 & 0 & 0,1667 & 0 & 0 & 0 & 0 & 0 & 0 & 0 & 0 & 0 & 0,1752 & 0 & 0 & 0 & 0 \\
\hline Capitaliz & 0 & 0 & 0 & 0 & 0 & 0 & 0 & 0,5 & 0 & 0 & 0 & 0 & 0 & 0 & 0 & 0,0866 & 0 & 0,6667 & 0 & 0,2277 & 70,2291 & 0,4364 & 0 & 0 \\
\hline Innovat & 0,1008 & 0,0857 & 0 & 0 & 0 & 0,1667 & 0 & 0 & 0,3333 & 0,0833 & 0 & 0 & 0 & 0 & 0 & 0,0433 & 0,1143 & 0 & 0,2546 & 0 & 0,0764 & 0,1091 & 0,4815 & 0,4331 \\
\hline Output & 0,2016 & 0,1714 & 0 & 0 & 0 & 0,1667 & 0 & 0 & 0,6667 & 0 & 0 & 0 & 0 & 0 & 0 & 0,28 & 0,2286 & 0 & 0,5092 & 0,1139 & 0 & 0,2182 & 0 & 0 \\
\hline Profit & 0 & 0 & 0 & 0 & 0 & 0 & 0 & 0 & 0 & 0,25 & 0 & 0 & 0 & 0 & 0 & 0,1616 & 0,2286 & 0 & 0 & 0,1139 & 0,4582 & 0 & 0 & 0 \\
\hline $\begin{array}{l}\text { Costs } \\
\text { New }\end{array}$ & 0,0573 & 0,0365 & 0 & 0 & 0 & 0,0833 & 0 & 0 & 0 & 0 & 0 & 0 & 0 & 0 & 0 & 0 & 0 & 0 & 0 & 0,1057 & 0 & 0 & 0 & 0,4664 \\
\hline Tech & 0 & 0,0122 & 0 & 0 & 0 & 0,25 & 0 & 0 & 0 & 0,3333 & 0 & 0 & 0 & 0 & 0 & 0 & 0 & 0 & 0 & 0,0352 & 0 & 0 & 0,5186 & 0 \\
\hline
\end{tabular}


Table 4. Limiting priorities for different network models.

\begin{tabular}{|c|c|c|c|c|c|c|c|c|}
\hline \multirow[b]{2}{*}{$\begin{array}{l}\text { Cluster } \\
\text { Name }\end{array}$} & \multirow[b]{2}{*}{ Node Name } & \multicolumn{3}{|c|}{ Network model (Figure 1) } & \multirow{2}{*}{$\begin{array}{c}\begin{array}{c}\text { Modified model } \\
\text { (Figure 4) }\end{array} \\
\text { Limiting } \\
\text { Priorities }\end{array}$} & \multicolumn{3}{|c|}{ Inverted task (Figure 5) } \\
\hline & & $\begin{array}{l}\text { Limiting } \\
\text { Priorities }\end{array}$ & $\begin{array}{c}\text { Priorities, } \\
\text { Normalized } \\
\text { by Cluster }\end{array}$ & $\begin{array}{l}\text { Sum in } \\
\text { Cluster }\end{array}$ & & $\begin{array}{l}\text { Limiting } \\
\text { Priorities }\end{array}$ & $\begin{array}{c}\text { Priorities, } \\
\text { Normalized } \\
\text { by Cluster }\end{array}$ & $\begin{array}{l}\text { Sum in } \\
\text { Cluster }\end{array}$ \\
\hline \multirow{4}{*}{ Banks } & Assets & 0,0225 & 0,3479 & \multirow{4}{*}{0,0647} & 0,0225 & 0,0722 & 0,2150 & \multirow{4}{*}{0,3358} \\
\hline & Credits & 0,019 & 0,2933 & & 0,019 & 0,0877 & 0,2612 & \\
\hline & Deposits & 0,0109 & 0,1687 & & 0,0109 & 0,0606 & 0,1806 & \\
\hline & Mortgage & 0,0123 & 0,1901 & & 0,0123 & 0,1153 & 0,3432 & \\
\hline \multirow{6}{*}{ Government } & $\begin{array}{l}\text { Export } \\
\text { Incentive }\end{array}$ & 0 & 0 & \multirow{6}{*}{0} & & 0 & 0 & \multirow{6}{*}{0,3647} \\
\hline & $\begin{array}{l}\text { Financial } \\
\text { Support }\end{array}$ & 0 & 0 & & & 0,1686 & 0,4624 & \\
\hline & Interest Rate & 0 & 0 & & & 0,0522 & 0,14318 & \\
\hline & $\begin{array}{l}\text { Poor Assets } \\
\text { Purchase }\end{array}$ & 0 & 0 & & & 0,0825 & 0,2261 & \\
\hline & Protectionism & 0 & 0 & & & 0,0148 & 0,0405 & \\
\hline & Tax Cut & 0 & 0 & & & 0,0466 & 0,1278 & \\
\hline \multirow{5}{*}{ Macrofactors } & $\begin{array}{l}\text { Capital } \\
\text { Investments }\end{array}$ & 0,0183 & 0,1003 & \multirow{5}{*}{0,1826} & 0,0183 & 0 & 0 & \multirow{5}{*}{0} \\
\hline & Employment & 0,0292 & 0,1601 & & 0,0292 & 0 & 0 & \\
\hline & Inflation & 0,0085 & 0,0468 & & 0,0085 & 0 & 0 & \\
\hline & Market Shares & 0,0145 & 0,0796 & & 0,0145 & 0 & 0 & \\
\hline & $\begin{array}{l}\text { Production } \\
\text { Level }\end{array}$ & 0,112 & 0,6132 & & 0,112 & 0 & 0 & \\
\hline \multirow{3}{*}{ Population } & Demand & 0,0179 & 0,3368 & \multirow{3}{*}{0,0531} & 0,0179 & 0,0448 & 0,4165 & \multirow{3}{*}{0,1075} \\
\hline & Incomes & 0,0013 & 0,0253 & & 0,0013 & 0,0459 & 0,4269 & \\
\hline & Trust & 0,0339 & 0,6379 & & 0,0339 & 0,0168 & 0,1566 & \\
\hline \multirow{4}{*}{ Producers } & Capitalization & 0,1866 & 0,2946 & \multirow{4}{*}{0,6334} & 0,1866 & 0,0106 & 0,085 & \multirow{4}{*}{0,1251} \\
\hline & Innovation & 0,1354 & 0,2138 & & 0,1354 & 0,0775 & 0,6192 & \\
\hline & Output & 0,1849 & 0,292 & & 0,1849 & 0,0106 & 0,0844 & \\
\hline & Profit & 0,1264 & 0,1996 & & 0,1264 & 0,0264 & 0,2114 & \\
\hline \multirow{2}{*}{$\begin{array}{c}\text { Science \& } \\
\text { Technologies }\end{array}$} & Costs & 0,0367 & 0,5552 & \multirow[b]{2}{*}{0,0661} & 0,0367 & 0,0251 & 0,3754 & \multirow[b]{2}{*}{0,0669} \\
\hline & $\begin{array}{l}\text { New } \\
\text { Technologies }\end{array}$ & 0,0294 & 0,4448 & & 0,0294 & 0,0418 & 0,6246 & \\
\hline
\end{tabular}


Table 5. Unweighted supermatrix for the inverted network model.

\begin{tabular}{|c|c|c|c|c|c|c|c|c|c|c|c|c|c|c|c|c|c|c|c|c|c|c|c|c|}
\hline & $\begin{array}{l}n \\
0 \\
0 \\
0 \\
0\end{array}$ & $\begin{array}{l}: \stackrel{\mathscr{Z}}{0} \\
\stackrel{0}{0}\end{array}$ & $\begin{array}{l}\stackrel{0}{=} \\
0 \\
0 \\
00 \\
0\end{array}$ & 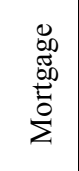 & 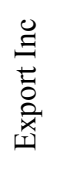 & 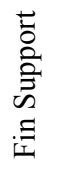 & 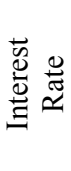 & 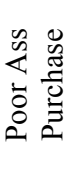 & 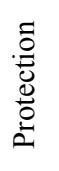 & 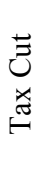 & 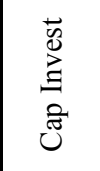 & 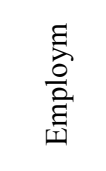 & 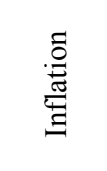 & 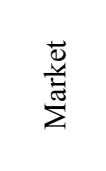 & 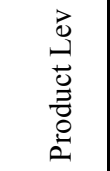 & 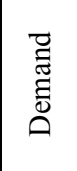 & 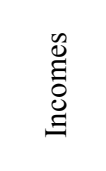 & $\underset{E}{\vec{E}}$ & 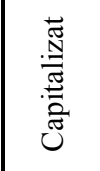 & 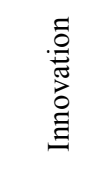 & 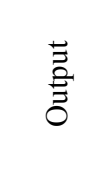 & $\begin{array}{l}\text { 릉 } \\
\text { D. }\end{array}$ & $\begin{array}{l}\frac{n}{0} \\
\dot{0} \\
0\end{array}$ & 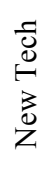 \\
\hline Assets & 0 & 0,4 & 0,3333 & 0,5 & 0 & 0 & 0 & 0 & 0 & 0 & 0,4 & 0,4 & 0 & 0 & 0,2 & 0 & 0 & 0 & 0 & 0,5 & 0,25 & 0 & 0 & 0 \\
\hline Credits & 0,25 & 0 & 0 & 0 & 0 & 0 & 0 & 0 & 0 & 0 & 0 & 0 & 0 & 0,25 & 0,6 & 0,75 & 0,3333 & 0,5 & 0 & 0,5 & 0,75 & 0 & 1 & 1 \\
\hline Deposits & 0,5 & 0,4 & 0 & 0,5 & 0 & 0 & 0 & 0 & 0 & 0 & 0,2 & 0,2 & 0 & 0,5 & 0,2 & 0 & 0 & 0 & 0 & 0 & 0 & 0 & 0 & 0 \\
\hline Mortgage & 0,25 & 0,2 & 0,6667 & 0 & 0 & 0 & 0 & 0 & 0 & 0 & 0,4 & 0,4 & 0 & 0,25 & 0 & 0,25 & 0,6667 & 0,5 & 0 & 0 & 0 & 0 & 0 & 0 \\
\hline $\begin{array}{l}\text { Export } \\
\text { Inc }\end{array}$ & 0 & 0 & 0 & 0 & 0 & 0 & 0 & 0 & 0 & 0 & 0 & 0 & 0 & 0 & 0 & 0 & 0 & 0 & 0 & 0 & 0 & 0 & 0 & 0 \\
\hline Fin Sup & 0 & 0,6667 & 0 & 0,75 & 0 & 0 & 0 & 0 & 0 & 0 & 0 & 0 & 0 & 0 & 0 & 0 & 0 & 0 & 0 & 0,3333 & 0,3333 & 0 & 1 & 1 \\
\hline Interest & 0 & 0,3333 & 0 & 0,25 & 0 & 0 & 0 & 0 & 0 & 0 & 0 & 0 & 0 & 0 & 0 & 0 & 0 & 0 & 0 & 0 & 0 & 0 & 0 & 0 \\
\hline Poor Ass & 1 & 0 & 0 & 0 & 0 & 0 & 0 & 0 & 0 & 0 & 0 & 0 & 0 & 0 & 0 & 0 & 0 & 0 & 1 & 0 & 0 & 0 & 0 & 0 \\
\hline Protect & 0 & 0 & 0 & 0 & 0 & 0 & 0 & 0 & 0 & 0 & 0 & 0 & 0 & 0 & 0 & 0 & 0 & 0 & 0 & 0,3333 & 0,6667 & 0 & 0 & 0 \\
\hline Tax Cut & 0 & 0 & 0 & 0 & 0 & 0 & 0 & 0 & 0 & 0 & 0 & 0 & 0 & 0 & 0 & 0 & 1 & 1 & 0 & 0,3333 & 0 & 1 & 0 & 0 \\
\hline $\begin{array}{c}\text { Cap } \\
\text { Invest }\end{array}$ & 0 & 0 & 0 & 0 & 0 & 0 & 0 & 0 & 0 & 0 & 0 & 0 & 0 & 0 & 0 & 0 & 0 & 0 & 0 & 0 & 0 & 0 & 0 & 0 \\
\hline Employ & 0 & 0 & 0 & 0 & 0 & 0 & 0 & 0 & 0 & 0 & 0 & 0 & 0 & 0 & 0 & 0 & 0 & 0 & 0 & 0 & 0 & 0 & 0 & 0 \\
\hline Inflation & 0 & 0 & 0 & 0 & 0 & 0 & 0 & 0 & 0 & 0 & 0 & 0 & 0 & 0 & 0 & 0 & 0 & 0 & 0 & 0 & 0 & 0 & 0 & 0 \\
\hline Market & 0 & 0 & 0 & 0 & 0 & 0 & 0 & 0 & 0 & 0 & 0 & 0 & 0 & 0 & 0 & 0 & 0 & 0 & 0 & 0 & 0 & 0 & 0 & 0 \\
\hline Prod Lev & 0 & 0 & 0 & 0 & 0 & 0 & 0 & 0 & 0 & 0 & 0 & 0 & 0 & 0 & 0 & 0 & 0 & 0 & 0 & 0 & 0 & 0 & 0 & 0 \\
\hline Demand & 0 & 0,6483 & 0 & 0,5 & 0 & 0 & 0 & 0 & 0 & 0 & 0 & 0 & 0 & 0,3333 & 0,6667 & 0 & 0 & 0 & 1 & 0,5 & 0,75 & 0,75 & 0 & 0 \\
\hline Incomes & 0 & 0,2297 & 0,75 & 0,25 & 0 & 0 & 0 & 0 & 0 & 0 & 0 & 0 & 0 & 0,6667 & 0,3333 & 0 & 0 & 0 & 0 & 0,5 & 0,25 & 0,25 & 0 & 0 \\
\hline Trust & 0 & 0,122 & 0,25 & 0,25 & 0 & 0 & 0 & 0 & 0 & 0 & 0 & 0 & 0 & 0 & 0 & 0 & 0 & 0 & 0 & 0 & 0 & 0 & 0 & 0 \\
\hline Capitaliz & 0 & 0 & 0 & 0 & 0 & 0 & 0 & 0 & 0 & 0 & 0 & 0 & 0 & 0 & 0 & 0 & 0 & 0 & 0 & 0,2857 & 0,1429 & 0 & 0 & 0 \\
\hline Innovat & 0 & 0 & 0 & 0 & 0 & 0 & 0 & 0 & 0 & 0 & 0,3333 & 0,3333 & 0,3333 & 0 & 0,1429 & 1 & 0 & 1 & 0,2857 & 0 & 0,2957 & 0,6667 & 1 & 1 \\
\hline Output & 0 & 0 & 0 & 0 & 0 & 0 & 0 & 0 & 0 & 0 & 0 & 0 & 0,6667 & 1 & 0,2857 & 0 & 0 & 0 & 0,1429 & 0,1429 & 0 & 0,3333 & 0 & 0 \\
\hline Profit & 0 & 0 & 0 & 0 & 0 & 0 & 0 & 0 & 0 & 0 & 0,6667 & 0,6667 & 0 & 0 & 0,5715 & 0 & 0 & 0 & 0,5714 & 0,5714 & 0,5714 & 0 & 0 & 0 \\
\hline $\begin{array}{l}\text { Costs } \\
\text { New }\end{array}$ & 0 & 0 & 0 & 0 & 0 & 0 & 0 & 0 & 0 & 0 & 0 & 0 & 0 & 0 & 0 & 0 & 0 & 0 & 0 & 0 & 0 & 0 & 0 & 1 \\
\hline Tech & 0 & 0 & 0 & 0 & 0 & 0 & 0 & 0 & 0 & 0 & 1 & 1 & 1 & 0 & 1 & 0 & 0 & 0 & 0 & 1 & 1 & 1 & 1 & 0 \\
\hline
\end{tabular}


Table 6. Weighted supermatrix for the inverted network model.

\begin{tabular}{|c|c|c|c|c|c|c|c|c|c|c|c|c|c|c|c|c|c|c|c|c|c|c|c|c|}
\hline & $\begin{array}{l}0 \\
0 \\
0 \\
0 \\
2\end{array}$ & 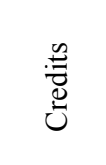 & 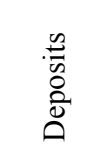 & 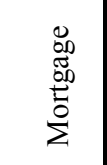 & 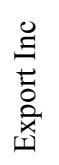 & 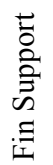 & 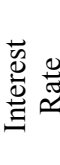 & 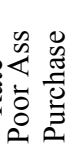 & 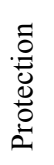 & 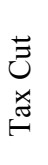 & 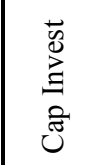 & 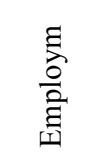 & 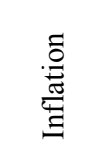 & 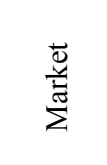 & 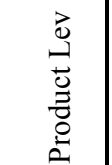 & 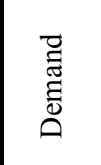 & 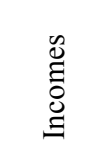 & $\underset{E}{\vec{E}}$ & 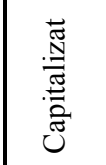 & $\begin{array}{l}\text {.0 } \\
\text { 壳 } \\
\text { ठ } \\
\Xi \\
\Xi\end{array}$ & 壳 & $\begin{array}{l}\overrightarrow{0} \\
0 \\
0 \\
0\end{array}$ & 品 & $\begin{array}{l}\frac{0}{0} \\
\stackrel{0}{-1} \\
3 \\
z \\
z\end{array}$ \\
\hline Assets & 0 & 0,1143 & 0,2222 & 0,1429 & 0 & 0 & 0 & 0 & 0 & 0 & 0,113 & 0 & 0 & 0 & 0,052 & 0 & 0 & 0 & 0 & 0,0714 & 0,0357 & 0 & 0 & 0 \\
\hline Credits & 0,0833 & 0 & 0 & 0 & 0 & 0 & 0 & 0 & 0 & 0 & 0 & 0 & 0,0755 & 0 & 0,1559 & 0,4838 & 0,2559 & 0,2698 & 0 & 0,0714 & 0,1072 & 0 & 0,0762 & 0,0762 \\
\hline Deposits & 0,1667 & 0,1143 & 0 & 0,1429 & 0 & 0 & 0 & 0 & 0 & 0 & 0,0565 & 0 & 0,151 & 0 & 0,052 & 0 & 0 & 0 & 0 & 0 & 0 & 0 & 0 & 0 \\
\hline Mortgage & 0,0833 & 0,0571 & 0,4444 & 0 & 0 & 0 & 0 & 0 & 0 & 0 & 0,113 & 0 & 0,0755 & 0 & 0 & 0,1613 & 0,5117 & 0,2698 & 0 & 0 & 0 & 0 & 0 & 0 \\
\hline $\begin{array}{c}\text { Export } \\
\text { Inc }\end{array}$ & 0 & 0 & 0 & 0 & 0 & 0 & 0 & 0 & 0 & 0 & 0 & 0 & 0 & 0 & 0 & 0 & 0 & 0 & 0 & 0 & 0 & 0 & 0 & 0 \\
\hline Fin Sup & 0 & 0,381 & 0 & 0,4286 & 0 & 0 & 0 & 0 & 0 & 0 & 0 & 0 & 0 & 0 & 0 & 0 & 0 & 0 & 0 & 0,0952 & 0,0952 & 0 & 0,238 & 0,238 \\
\hline Interest & 0 & 0,1905 & 0 & 0,1429 & 0 & 0 & 0 & 0 & 0 & 0 & 0 & 0 & 0 & 0 & 0 & 0 & 0 & 0 & 0 & 0 & 0 & 0 & 0 & 0 \\
\hline Poor Ass & 0,6667 & 0 & 0 & 0 & 0 & 0 & 0 & 0 & 0 & 0 & 0 & 0 & 0 & 0 & 0 & 0 & 0 & 0 & 0,4 & 0 & 0 & 0 & 0 & 0 \\
\hline Protect & 0 & 0 & 0 & 0 & 0 & 0 & 0 & 0 & 0 & 0 & 0 & 0 & 0 & 0 & 0 & 0 & 0 & 0 & 0 & 0,0952 & 0,1905 & 0 & 0 & 0 \\
\hline Tax Cut & 0 & 0 & 0 & 0 & 0 & 0 & 0 & 0 & 0 & 0 & 0 & 0 & 0 & 0 & 0 & 0 & 0,2325 & 0,1634 & 0 & 0,0952 & 0 & 0,3333 & 0 & 0 \\
\hline $\begin{array}{c}\text { Cap } \\
\text { Invest }\end{array}$ & 0 & 0 & 0 & 0 & 0 & 0 & 0 & 0 & 0 & 0 & 0 & 0 & 0 & 0 & 0 & 0 & 0 & 0 & 0 & 0 & 0 & 0 & 0 & 0 \\
\hline Employ & 0 & 0 & 0 & 0 & 0 & 0 & 0 & 0 & 0 & 0 & 0 & 0 & 0 & 0 & 0 & 0 & 0 & 0 & 0 & 0 & 0 & 0 & 0 & 0 \\
\hline Inflation & 0 & 0 & 0 & 0 & 0 & 0 & 0 & 0 & 0 & 0 & 0 & 0 & 0 & 0 & 0 & 0 & 0 & 0 & 0 & 0 & 0 & 0 & 0 & 0 \\
\hline Market & 0 & 0 & 0 & 0 & 0 & 0 & 0 & 0 & 0 & 0 & 0 & 0 & 0 & 0 & 0 & 0 & 0 & 0 & 0 & 0 & 0 & 0 & 0 & 0 \\
\hline Prod Lev & 0 & 0 & 0 & 0 & 0 & 0 & 0 & 0 & 0 & 0 & 0 & 0 & 0 & 0 & 0 & 0 & 0 & 0 & 0 & 0 & 0 & 0 & 0 & 0 \\
\hline Demand & 0 & 0,0926 & 0 & 0,0714 & 0 & 0 & 0 & 0 & 0 & 0 & 0 & 0 & 0,0313 & 0,0818 & 0,0539 & 0 & 0 & 0 & 0,2 & 0,0714 & 0,1071 & 0,125 & 0 & 0 \\
\hline Incomes & 0 & 0,0328 & 0,25 & 0,0357 & 0 & 0 & 0 & 0 & 0 & 0 & 0 & 0 & 0,0626 & 0,0273 & 0,0269 & 0 & 0 & 0 & 0 & 0,0714 & 0,0357 & 0,0417 & 0 & 0 \\
\hline Trust & 0 & 0,0174 & 0,0833 & 0,0357 & 0 & 0 & 0 & 0 & 0 & 0 & 0 & 0 & 0 & 0 & 0 & 0 & 0 & 0 & 0 & 0 & 0 & 0 & 0 & 0 \\
\hline Capitaliz & 0 & 0 & 0 & 0 & 0 & 0 & 0 & 0 & 0 & 0 & 0 & 0 & 0 & 0 & 0 & 0 & 0 & 0 & 0 & 0,0816 & 0,0408 & 0 & 0 & 0 \\
\hline Innovat & 0 & 0 & 0 & 0 & 0 & 0 & 0 & 0 & 0 & 0 & 0,1884 & 0,2626 & 0 & 0,5264 & 0,0742 & 0,355 & 0 & 0,297 & 0,1143 & 0 & 0,0816 & 0,2222 & 0,304 & 0,304 \\
\hline Output & 0 & 0 & 0 & 0 & 0 & 0 & 0 & 0 & 0 & 0 & 0 & 0,5252 & 0,604 & 0,1755 & 0,1484 & 0 & 0 & 0 & 0,0571 & 0,0408 & 0 & 0,1111 & 0 & 0 \\
\hline Profit & 0 & 0 & 0 & 0 & 0 & 0 & 0 & 0 & 0 & 0 & 0,3768 & 0 & 0 & 0 & 0,2969 & 0 & 0 & 0 & 0,2286 & 0,1633 & 0,1633 & 0 & 0 & 0 \\
\hline Costs & 0 & 0 & 0 & 0 & 0 & 0 & 0 & 0 & 0 & 0 & 0 & 0 & 0 & 0 & 0 & 0 & 0 & 0 & 0 & 0 & 0 & 0 & 0 & 0,3818 \\
\hline $\begin{array}{l}\text { New } \\
\text { Tech }\end{array}$ & 0 & 0 & 0 & 0 & 0 & 0 & 0 & 0 & 0 & 0 & 0,1523 & 0,2122 & 0 & 0,1891 & 0,14 & 0 & 0 & 0 & 0 & 0,1429 & 0,1429 & 0,1667 & 0,3818 & 0 \\
\hline
\end{tabular}



Table 7. Cluster matrix for the inverted network.

\begin{tabular}{|c|c|c|c|c|c|c|}
\hline $\begin{array}{c}\text { Cluster } \\
\text { Name }\end{array}$ & Banks & Government & Macrofactors & Population & Producers & $\begin{array}{c}\text { Science \& } \\
\text { Technologies }\end{array}$ \\
\hline Banks & 0,2857 & 0 & 0,2598 & 0,5396 & 0,1429 & 0,0762 \\
\hline Government & 0,5714 & 0 & 0 & 0,1634 & 0,2857 & 0,238 \\
\hline Macrofactors & 0 & 0 & 0 & 0 & 0 & 0 \\
\hline Population & 0,1429 & 0 & 0,0808 & 0 & 0,1429 & 0 \\
\hline Producers & 0 & 0 & 0,5195 & 0,297 & 0,2857 & 0,3041 \\
\hline $\begin{array}{c}\text { Science \& } \\
\text { Technologies }\end{array}$ & 0 & 0 & 0,14 & 0 & 0,1429 & 0,3818 \\
\hline
\end{tabular}

\section{REFERENCES}

Saaty, T.L. (2001). Decision making with dependence and feedback: The analytic network process. Pittsburgh, PA: RWS Publications.

SuperDecisions software (2009). Software for the ANP, downloaded from the website http://www.superdecisions.com. 